\title{
Condition Monitoring and Fault Diagnosis for an Antifalling Safety Device
}

\author{
Guangxiang Yang ${ }^{1,2}$ and Hua Liang ${ }^{1}$ \\ ${ }^{1}$ Chongqing Engineering Laboratory for Detection, Control and Integrated System, Chongqing Technology and Business University, \\ Chongqing 400067, China \\ ${ }^{2}$ Research Center of System Health Maintenance, Chongqing Technology and Business University, Chongqing 400067, China
}

Correspondence should be addressed to Guangxiang Yang; ygxmonkey@126.com

Received 7 April 2015; Accepted 26 May 2015

Academic Editor: Chuan Li

Copyright (C) 2015 G. Yang and H. Liang. This is an open access article distributed under the Creative Commons Attribution License, which permits unrestricted use, distribution, and reproduction in any medium, provided the original work is properly cited.

\begin{abstract}
There is a constant need for the safe operation and reliability of antifalling safety device (AFSD) of an elevator. This paper reports an experimental study on rotation speed and catching torque monitoring and fault diagnosis of an antifalling safety device in a construction elevator. Denoising the signal using wavelet transform is presented in this paper. Based on the denoising effects for several types of wavelets, the sym8 wavelet basis, which introduces the high order approximation and an adaptive threshold, is employed for denoising the signal. The experimental result shows a maximum data error reduction of 7.5\% is obtained and SNRs (signal-to-noise ratio) of rotation speed and catching torque are improved for $3.9 \%$ and $6.4 \%$, respectively.
\end{abstract}

\section{Introduction}

The construction hoist is one of the essential mechanical devices in the construction. With flourish development of construction industry in China, higher demands are also asked for the performance and reliability of the construction hoist. A construction elevator is widely used in tall building construction and some civil engineering fields such as bridge construction, underground construction, large chimney construction, and shipbuilding.

A construction hoist is equipped with an antifalling safety device to keep the unexpected falling and ensure the safe operation of the elevator. An antifalling safety device is the most important safety device in the pinion and rack builder's hoist, which can prevent the cage running over speed and effectively reduce the cage dropping accident. It is a kind of velocity controlled safety device which prevents the dropping of the elevator cage mounted on rack rails. When the speed of elevator cage reaches the triggering speed of antifalling safety device, the compression spring in the safety device is compressed and the braking force is increased by the friction of cone drum braking surface which is in contact with the brake band. With this braking force increasing, the elevator cage is braked and stopped on the rack rails $[1,2]$. The motor power and the circuits of controller of elevator are cut off as soon as the antifalling safety device is triggered to action. An antifalling safety device is one typical model of building hoist gear cone drum progressive antifalling safety device and is designed for prevent building hoist cages or counterweight from falling. It is made up of gear, centrifugal speed limiter device, cone drum, and brake device. An antifalling safety device is shown in Figure 1.

An antifalling safety device is composed of the following components: a pinion, a friction plate, a centrifugal block, an outer cone, a disc spring, a microswitch, a screw nut, and a spring.

When the hoist cage is speeding down, the rotation speed of pinion is increasing and the centrifugal block rotated with pinion at the same speed is thrown outwards and meshed in the outer cone. This makes the disc spring compressed and the positive pressure between the outer cone and the friction plate is increased gradually. Hence, the torque is produced. Catching torque is increased with the catching process to achieve gradual braking, until the cage is stopped. The construction elevator related specifications for an antifalling safety device in China including JG121-2000 


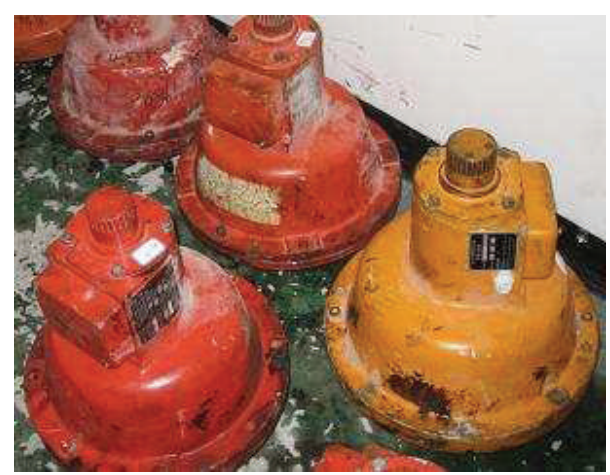

FIgURE 1: An antifalling safety device.

building hoist, pinion and cone progressive type safety device, GB10055-2007 construction hoist safety regulations, and GB/T10054-2005 builder's hoist. These documents specify the operation, maintenance, and verification. For example, it is strictly pointed out in GB10055-2007 that the valid calibration deadline is one year; the tripping speed and catching distance of must meet the requirement of GB/T10054-2005.

According to the reports from a special laboratory of Liaoning province in China [3], there are 143 unqualified or failed antifalling safety devices in totally 1428 detected antifalling safety device in 2012 . The unqualified rate reached $10 \%$ and in which $90 \%$ of the faults are due to rotation speed fault, the other is mainly the failure of braking distance and braking load. This fully demonstrates the importance and necessity of antifalling safety device of regular inspection.

Current research on the antifalling safety device mainly concentrated in the aspects of design, safety verification, and structural analysis. The condition monitoring and fault diagnosis scheme are rarely reported. Furthermore, the detected data used to calculate the catching distance and the maximum catching load of antifalling safety device is often contaminated or influenced by the electromagnetic interference. Therefore the accuracy and the reliability of data have to be improved.

In this paper, a condition monitoring system is developed and a fault diagnosis scheme is presented by using the wavelet transform thresholding denoising method. By reviewing the state-of-the-art in wavelet for rotating machine condition monitoring, the paper discusses specific needs of an antifalling safety device monitoring system addressed by wavelet, describes features of wavelet, and investigates the experiment study of wavelet in an antifalling safety device monitoring system. On the basis of comparison of denoising effect for several types of wavelets, the sym8 wavelet basis, which introduces the high order approximation, tuned scale factors, and an adaptive threshold, is employed for denoising the original catching torque and rotation speed signals. Experimental results show that the denoised data is smoother and the reliability of signal is improved. The sym 8 wavelet with thresholding is proven to be better than the others. The average error of pulse peak data is reduced by $7.5 \%$ when the sym 8 wavelet is applied for denoising the observed signal which proves the effectiveness and reliability of this method.

\section{Related Works}

An antifalling safety device in nature is a kind of rotating machinery. Rotating machine analysis has been conducted for many decades [4]. It is becoming popular for on-going production condition based monitoring system. Rotating machines operating in production is greatly benefited from accurate performance data. For an antifalling safety device, Bai and Zhang present the system design of detection platform [5]. The condition of an antifalling safety device is monitored and the data is obtained by sensors with the detection platform. Therefore the original data is important for calibration and computation of the catching distance and the catching load. Li et al. [6] propose an intelligent diagnosis method for condition diagnosis of rotating machinery by using wavelet transform and ant colony optimization, in order to detect faults and distinguish fault types at an early stage. Venugopal et al. [7] present a signal processing technique for machine performance monitoring which exploits fluctuations in phase angles of machine rotational frequency signals to determine their dynamic temporal coherence. Sun et al. [8] propose a fault diagnosis method for rotating machinery using ant colony optimization and possibility theory. Mueller and Pratt [9] present the theory and practical application for using radio frequency polarimetry in analyzing rotating machines. A neural network-based fault detection and isolation scheme is presented to detect and isolate faults in a highly nonlinear dynamics of an aircraft jet engine by TayaraniBathaie et al. [10]. Yang et al. [11] present a generalization of multidimensional linear regression to facilitate multisensor fault detection and signal reconstruction through the use of analytical optimization.

However, as a rotating machine, the antifalling safety device is more easily to be verified on condition that the original data are not noised. The most key problem is the confused detected result by the noised signal. These signals are used for calibration and parameter calculation such as catching distance and the catching load of a safety device. There are few researches of detected signals and data reported in the past. For the noised signal, an efficient technique for signal processing is the wavelet transform.

The wavelet transform, a signal processing technique based on a windowing approach of dilated "scaled" and shifted wavelets, is being applied to a broad range of engineering applications and other applications such as coding, data compression, structural detection and noise reduction, and spectroscopy signal processing [12-15]. Wavelet transform is also widely used in machinery diagnostics and measurement $[16,17]$.

Dziedziech et al. [18] present the time-variant frequency response function-based on the continuous wavelet transform for the analysis of time-variant systems. The focus is on the comparative study of various broadband input excitations. Measurement of groove race defect width in thrust bearing is presented by processing vibration signal using wavelet based technique by Singh and Kumar [19]. Wavelet features are computed using discrete wavelet transform (DWT) from the vibration signals of centrifugal pumps by Muralidharan and Sugumaran [20]. The seismic damage 


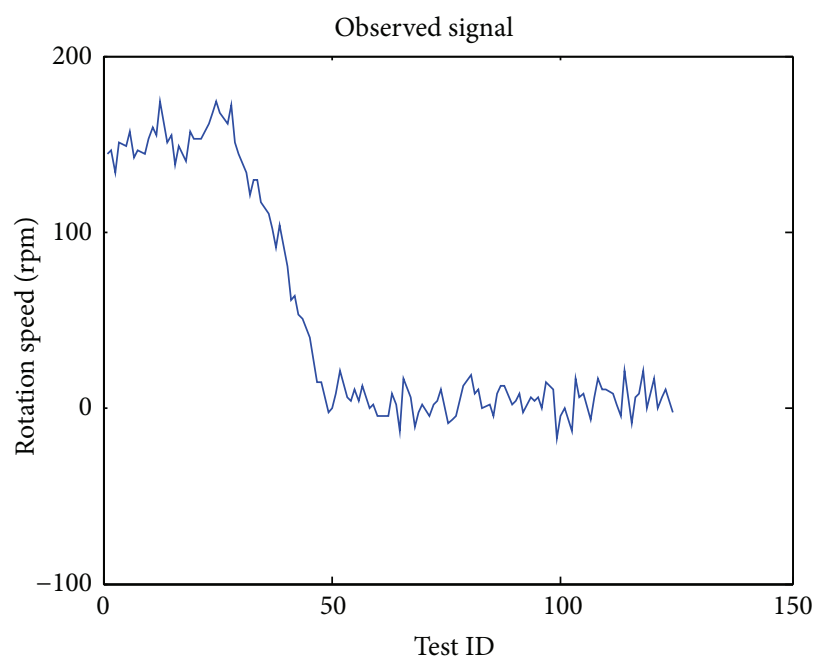

(a)

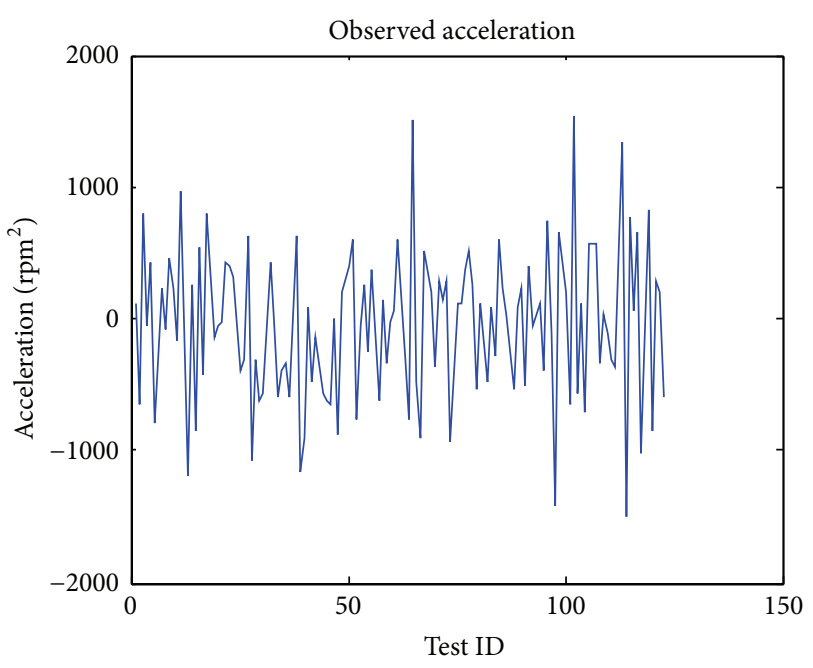

(b)

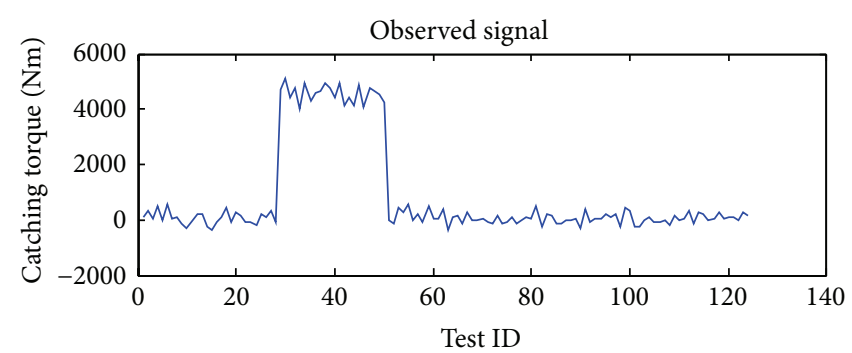

(c)

FIgURE 2: Observed signal of an antifalling safety device.

level of a structure is assessed using damage indices and the continuous wavelet transformation (CWT) technique is applied to compute the dissipated energy during ground shaking by Rajeev and Wijesundara [21]. Li et al. [22] review some important developments in TDLAS (tunable diode laser absorption spectroscopy), from its basic principles as a spectroscopic tool to the demonstration of gas absorption measurements, emphasizing signal enhancement and noise reduction techniques developed for improving current TDLAS performance. A recursive algorithm [23] is presented to estimate the variance of the noise and prove its convergence and investigate its mathematical properties. All of these works have greatly improved the research and application of wavelet transform.

In this paper, thresholding in wavelet domain is used for noise reduction of rotation speed and catching torque in antifalling safety device diagnosis. The proposed signal denoising method in this paper is based on the method of Johnstone and Donoho. It has been proven that this method has been successfully applied to a wide class of nonstationary signals. The signal denoising method is successfully applied in the measurement of safety device in this paper. The denoised signal is smoother and the noised data is reduced, which guarantee the accuracy and reliability of an antifalling safety device.

\section{Fault Diagnosis with Wavelet Transform}

Because of the environment interface, the signal of an antifalling safety device is more easily to be noised. The typical originals are shown in Figure 2.

In Figure 2, "test ID" means the data sequence number when the signal is sampled from sensors. These signals are used for calibration and parameter calculation such as catching distance and the catching load of a safety device. Therefore the accuracy and reliability of acquired data may have a significant impact on the detection result of safety device. For the noised signal, an efficient technique for signal processing is the wavelet transform.

A novel approach for noise reduction has been established by Donoho and Johnstone in the 1990s [24]. It employs thresholding in the wavelet transformation and can be shown to be asymptotically near optimal for signals corrupted by additive white Gaussian noise (AWGN). This method has a wide variety of related problems such as linear inverse problems [25], data compression, and statistical estimation [26]. It is also used in construction, structure health monitoring [27], and civil engineering [28-31]. Noise reduction of wavelet transform has been proved to work well for a wide class of 1D and $2 \mathrm{D}$ signals. 
Given that finite samples $f_{i}$ of signal $f(t)$, which is overlaid with a white Gaussian noisy signal, is presented by:

$$
y_{i}=f_{i}+\sigma z_{i} ; \quad i=0,1,2, \ldots, n-1,
$$

where $z_{i}$ is a white Gaussian noise and $\sigma$ is the noise level.

For this continuous signal $f(t)$, its integral transformation is given by

$$
W_{f}(\alpha, \tau)=\int_{R} f(t) \overline{\psi_{\alpha \tau}(t)} d t=\left\langle f, \psi_{\alpha \tau}(t)\right\rangle .
$$

Equation (1) is a continuous wavelet transform (CWT) of $f(t)$, in which

$$
\psi_{\alpha \tau}(t)=\frac{1}{\sqrt{\alpha}} \psi\left(\frac{t-\tau}{\alpha}\right)
$$

is the wavelet basis function which is a group of functions scaled or shifted by a mother wavelet $\psi(t)$, where $\alpha$ and $\tau$ are scaling and shifting parameters of wavelet function $\psi(t)$, respectively.

The discrete wavelet function is given as

$$
\psi_{j, k}(t)=2^{-j / 2} \psi\left(2^{-j} t-k\right) ; \quad j, k \in Z,
$$

where $\psi(t)$ is the wavelet function and $2^{-j / 2} \psi\left(2^{-j} t-k\right)$ are scaled and shifted versions of $\psi(t)$ based on the values of scaling coefficient $j$ and shifting coefficient $k$, which is usually written as $\psi_{j, k}(t)$. The $j$ and $k$ coefficients take integer values for different scaling and shifted versions of $\psi(t)$.

In this case the continuous wavelet transform (CWT) of a continuous signal $f(t)(1)$ is given as

$$
D\left[W_{f}(j, k)\right]=2^{-j / 2} \int_{R} f(t) \overline{\psi\left(2^{-j} t-k\right)} d t
$$

and the discrete wavelet transform (DWT) of a discrete time sequence $f(t)$ can be derived:

$$
d_{j, k}=2^{-j / 2} \sum_{t} f(t) \psi\left(2^{-j} t-k\right) .
$$

Provided that $f_{k}$ is the discrete signal of $f(t), f_{k}=c_{0, k}$, the orthogonal wavelet decomposition formula [32] of signal $f(t)$ is computed as

$$
\begin{aligned}
c_{j, k} & =\sum_{n} c_{j-1, n} h_{n-2 k}, \\
d_{j, k} & =\sum_{n} c_{j-1, n} g_{n-2 k},
\end{aligned}
$$

$$
k=0,1,2, \ldots, N-1,
$$

where $c_{j, k}$ is the scale coefficient, $d_{j, k}$ is wavelet coefficient, $h, g$ is one pair of orthogonal mirror filters, $j$ is resolution level, and $N$ is the total number of data. The reconstruction of signal $f(t)$ is the reverse process of decomposition. The reconstruction equation is computed as

$$
c_{j-1, k}=\sum_{n} c_{j, n} h_{k-2 n}+\sum_{n} d_{j, n} g_{k-2 n} .
$$

The Donoho denoising scheme can recover the original signal $x_{i}$ from the noisy signal $y_{i}$ by the following steps.
TABLE 1: The calibrated tripping speed $(\mathrm{m} / \mathrm{s})$.

\begin{tabular}{lc}
\hline $\begin{array}{l}\text { Rated lifting speed of } \\
\text { construction hoist: } v\end{array}$ & $\begin{array}{c}\text { Calibrated tripping } \\
\text { speed of safety device: } v 1\end{array}$ \\
\hline$v \leq 0.60$ & $v 1 \leq 1.00$ \\
$0.60<v \leq 1.33$ & $v 1 \leq v+0.40$ \\
$v>1.33$ & $v 1 \leq 1.3 v$ \\
\hline
\end{tabular}

(1) Computing the orthogonal wavelet transform of noisy signal, selecting the proper wavelet and wavelet resolution level $j$. Based on (7), the noisy signal is decomposed to resolution level $j$ and the corresponding wavelet coefficient $d_{j, k}$ can be obtained.

(2) Computing the wavelet coefficient with soft thresholding and hard thresholding.

(3) Inverse wavelet transform. The original signal is obtained by thresholding with wavelet.

The thresholding feature of wavelet denoising is proved a more efficient method than conventional denoising methods [33]. The procedure starts by choosing an appropriate level for decomposition and decomposing the dynamics signal up to this level. For each level of decomposition, a certain threshold is selected and an appropriate thresholding criterion is applied. The threshold criterion can be either hard or soft thresholding. It is proved in this paper that denoising signal method using soft heuristic thresholding at level 4 by sym 8 wavelet is with an improvement of SNR (signalto-noise ratio). Symlets wavelet [34] is a function system and obtained by improving Daubechieds wavelet. Symlets wavelet is denoted as $\operatorname{sym} N(N=2,3, \ldots, 8)$, where $N$ is the order. Similar to Daubechieds wavelet, Symlets wavelet does not have specified algebraic expression. The catching torque signal and rotation speed signal measured on the detection platform is a real-world signal. This signal is particularly interesting because of noise introduced when a defect developed in the monitoring equipment during the measurement. Wavelet is used to smooth out and to remove the noised data of the measured signal. The obtained noiseless signals of catching torque and rotation speed are then reconstructed in time domain for diagnosis and calibration of antifalling safety device.

The detected data of an antifalling safety device including rotation speed and catching torque are often contaminated and noised by the environment electromagnetic interference. The data are key parameters to compute the tripping speed and the catching distance of an antifalling safety device, which is the criteria of whether it is qualified or not. Therefore, the detected signals of rotation speed and catching torque are denoised by thresholding with wavelet transform in this paper.

The calibrated tripping speed and the catching distance of an antifalling safety device are specified in the Chinese national specification for construction hoist, which are shown in Tables 1 and 2.

The rotation speed $s_{i}$ and the catching torque $t_{i}$ are measured on the detection platform of an antifalling platform. The sample size of two measured parameters is 125 which 


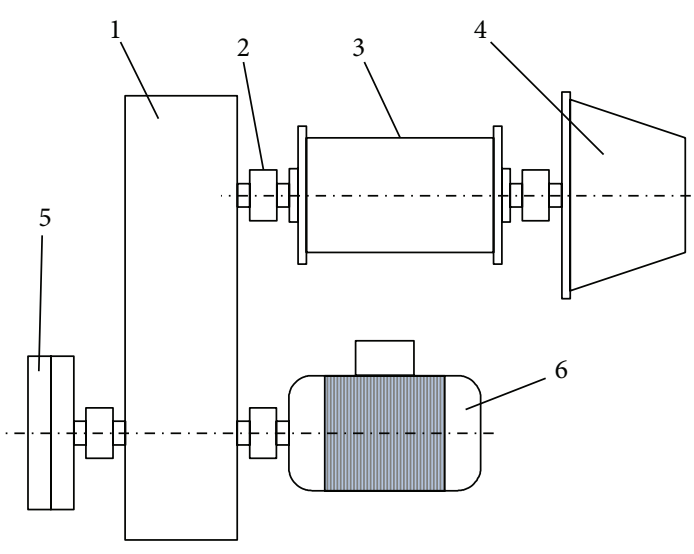

(a)

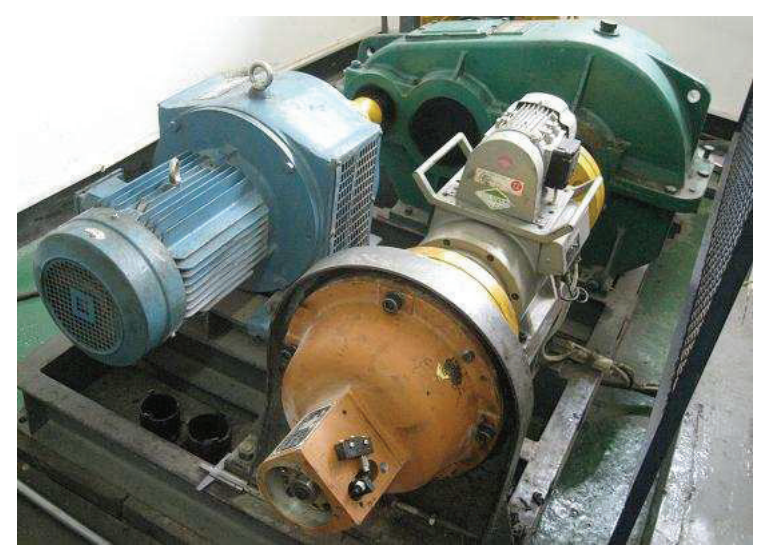

(b)

FIgURE 3: Experiment platform, where 1 is a gear box, 2 is a shaft coupling, 3 is sensor system of rotation speed and catching torque, 4 is an antifalling safety device, 5 is an electric motor, and 6 is an inertia load block.

TABLE 2: The catching distance.

\begin{tabular}{lc}
\hline $\begin{array}{l}\text { Specified lifting speed of } \\
\text { construction hoist: } v(\mathrm{~m} / \mathrm{s})\end{array}$ & $\begin{array}{c}\text { Specified catching distance } \\
\text { of safety device }(\mathrm{m})\end{array}$ \\
\hline$v \leq 0.65$ & $0.15-1.40$ \\
$0.65<v \leq 1.00$ & $0.25-1.60$ \\
$1.00<v \leq 1.33$ & $0.35-1.80$ \\
$v>1.33$ & $0.55-2.00$ \\
\hline
\end{tabular}

are obtained in 5 seconds. The wavelet transform sample size is 125 . The threshold value $[35,36]$ in the experiment is calculated by the following equation:

$$
\delta_{j}=\frac{\sigma \sqrt{2 \log N}}{\ln (e+j-1)}
$$

where $j$ is wavelet scale coefficient, $N$ is the sample size, $e$ is a constant coefficient 2.718 , and $\sigma$ is the standard deviation of the noise signal.

The rotation speed computation equation is given by

$$
v=\frac{(3.1415926 \times 8 \times 15 \times B)}{60000}
$$

in which $B$ is the measured rotation speed.

The catching distance is computed by the following formula:

catching distance $=\pi \times$ gear pitch diameter

$$
\times \frac{\text { compression of disc spring }}{\text { pitch }}
$$

in which, the gear pitch diameter and pitch is a fixed value; therefore, the catching distance is simplified as

$$
d=0.1885 \times D
$$

in which $D$ is the compression of disc spring.
TABLE 3: Parameters of SAJ40-1.2 antifalling safety device.

\begin{tabular}{lcc}
\hline Parameters & Units & Value \\
\hline Rated lifting speed & $\mathrm{m} / \mathrm{min}$ & 33 \\
Rated loading capacity & $\mathrm{kN}$ & 40 \\
Rated tripping speed & $\mathrm{m} / \mathrm{s}$ & 1.2 \\
Calibrated tripping speed & $\mathrm{m} / \mathrm{s}$ & 1 \\
Pinion gear modulus/teeth & $\mathrm{mm} /$ tooth & $8 / 15$ \\
Large nut pitch & $\mathrm{mm}$ & 2.0 \\
\hline
\end{tabular}

The catching load is computed by the following equation:

$$
M=\frac{(2 \times C)}{(1.5 \times 8 \times 15)},
$$

where $C$ is the measured catching torque.

\section{Experiments}

The condition monitoring of an antifalling safety device is conducted on a detection platform which is shown in Figure 3 . The pinion and centrifugal governor of safety device are mounted on the platform. The rotation speed of the pinion is adjusted to the calibrated tripping speed when the centrifugal governor starts working.

The antifalling safety device monitored in this experiment is SAJ40-1.2 type, which is produced in Shanghai. The parameters of this type of safety device are shown in Table 3.

A large number of antifalling safety devices have to be diagnosed every day since there are many construction enterprises in Chongqing, China. The antifalling safety devices to be detected are shown in Figure 4.

The safety device is mounted on the detection platform and is driven by a stepless speed regulation controller. When the safety device began to work, the rotation speed and the catching torque are sampled and stored in the computer. The data curve graph of these two parameters is shown on the computer screen. In the process of measurement, the nut 
TABLE 4: The denoised maximum rotation speed.

\begin{tabular}{lccl}
\hline $\begin{array}{l}\text { Observed } \\
\text { signal }\end{array}$ & $\begin{array}{c}\text { Denoised } \\
\text { signal }\end{array}$ & Reduction (\%) & Average reduction (\%) \\
\hline 173.8 & 156.2 & 11.3 & \\
168.7 & 158.7 & 6.3 & \\
167.2 & 155.1 & 7.8 & 7.5 \\
168.5 & 152.7 & 10.3 & \\
166.2 & 154.9 & 7.3 & \\
164.1 & 156.1 & 5.1 & \\
168.1 & 157.7 & 7.0 \\
169.6 & 154.6 & 9.7 & \\
159.2 & 151.3 & 5.2 \\
163.4 & 155.4 & 5.1 \\
\hline
\end{tabular}

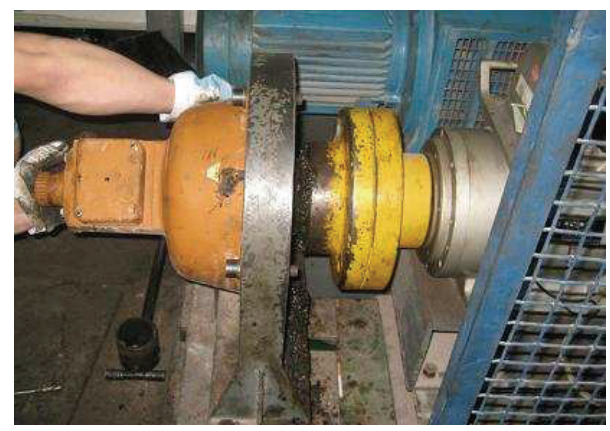

FIGURE 4: The antifalling safety devices to be detected.

and the pin cannot be reset but the centrifugal governor must be reset after each detecting. This measurement process repeated for 3 times until the maximum detected catching torque reaches or exceeds the computed torque. After 3 measurements, the nut and the pin are reset manually.

In the experiment, the compressed amount of disc spring, which is the length between the top of the pin and the small end cap plane, is recorded when the maximum catching torque reaches the required value in Chinese construction specification. The compressed length is used for the catching distance computation.

The rotation speed $s_{i}$ and the catching torque $t_{i}$ are measured on the detection platform and are transmitted to computer with a RS485 communication port. When received the data, they are denoised by thresholding with wavelet transform. The observed catching torque and the denoised catching torque are shown in Figure 5.

From Figure 5, it can be observed that there are some noisy signals on the two sides of the valid catching torque. The hard thresholding and soft thresholding noise reduction methods are applied in the data handling. The denoised catching torque signals with hard thresholding and soft thresholding are smoother than the observed signal. The original signal is the true signal or the expected output of the antifalling safety device based on its characteristics.

The observed signal of rotation speed is shown in Figure 6. The denoised rotation speed signals with hard thresholding and soft thresholding are shown in this figure, too. The rotation speed and acceleration of observed and denoised signals are shown in Figure 7.

According to the figures, it is shown that the wavelet transform denoising with thresholding is more suitable for the detecting. With the soft thresholding denoising, the maximum rotation speed of $173.8 \mathrm{rpm}$ is reduced to $156.2 \mathrm{rpm}$, which is $11.3 \%$ error reduction. There are 10 groups of data collected in Table 4, which shows an average reduction of maximum rotation speed is $7.5 \%$. The theoretical maximum rotation speed is a constant which is $156 \mathrm{rpm}$ based on the datasheet of detection platform.

Rotation speed and catching torque signal denoising algorithms are evaluated based on signal-to-noise ratio (SNR) and Mean Square Error (MSE) for obtaining low noise and readily observable sampling data. For simplicity, the results of rotation speed and catching torque data are partially listed. SNR $[37,38]$ of observed data and the denoised data is calculated in this experiment.

The SNR is given by the following equation [39]:

$$
\mathrm{SNR}=10 \lg \left[\frac{\sum_{i=1}^{N} s_{i}{ }^{2}}{\sum_{i=1}^{N}\left(s_{i}-g_{i}\right)^{2}}\right],
$$

where $s_{i}$ is the observed signal, $g_{i}$ is the denoised signal, and $N$ is the length of signal.

The MSE is estimated between the denoised signal and observed signal given by the following equation:

$$
\text { MSE }=\frac{1}{N} \sum_{i=1}^{N}\left(s_{i}-g_{i}\right)^{2},
$$

where $s_{i}$ is the observed signal, $g_{i}$ is denoised signal, and $N$ is the length of signal.

The detection platform aims to detect the rotation speed and the catching torque in which the falling of an elevator is simulated by a motor connected with safety device. The original rotation speed of the safety device is a motion with constant acceleration. The catching torque is theoretically a constant in each testing when the antifalling safety device is triggered to action.

The rotation speed is calculated by the following equation:

$$
v_{t}=v_{0}+\alpha t
$$

When an object moves with constant acceleration, $\alpha$ is the instantaneous acceleration at any point which is a constant, $v_{0}$ is the initial velocity, and $v_{t}$ is the velocity at any point.

According to Figure 7, the rotation speed of safety device has two sections, that is, acceleration and deceleration. Each testing point is sampled every 0.04 seconds. The acceleration phase is composed of 28 testing points which is $28 * 0.04 \mathrm{~s}-$ $0.04 \mathrm{~s}=1.08 \mathrm{~s}$. The deceleration section is composed of 24 testing points which consume 0.92 seconds. The original rotation speed at any testing point in acceleration and 
TABLE 5: The SNR results of rotation speed with hard thresholding and soft thresholding (dB).

\begin{tabular}{lcccccccccc}
\hline & Level 1 & Level 2 & Level 3 & Level 4 & Level 5 & Level 6 & Level 7 & Level 8 & Level 9 & Level 10 \\
\hline Hard thresholding & 26.51 & 26.53 & 26.95 & 26.89 & 27.02 & 26.97 & 26.11 & 26.43 & 26.64 & 26.12 \\
Soft thresholding & 26.35 & 27.10 & 27.04 & 27.17 & 27.13 & 26.91 & 27.01 & 26.88 & 27.10 & 26.73 \\
\hline
\end{tabular}

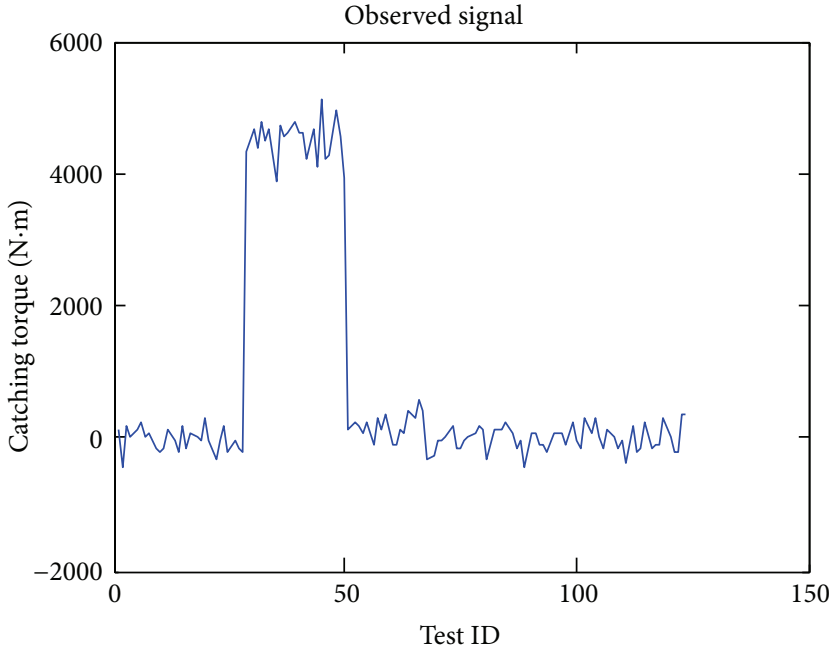

(a)

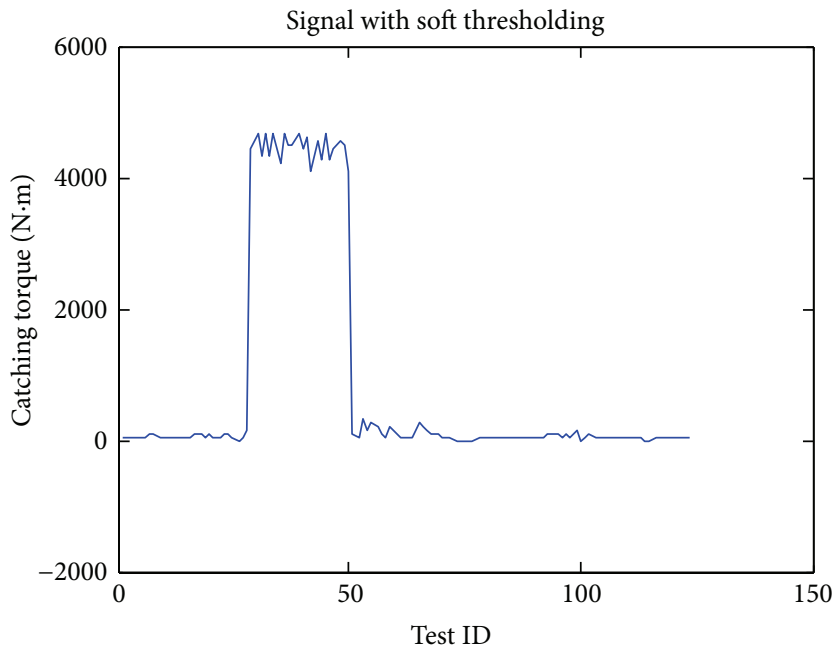

(c)

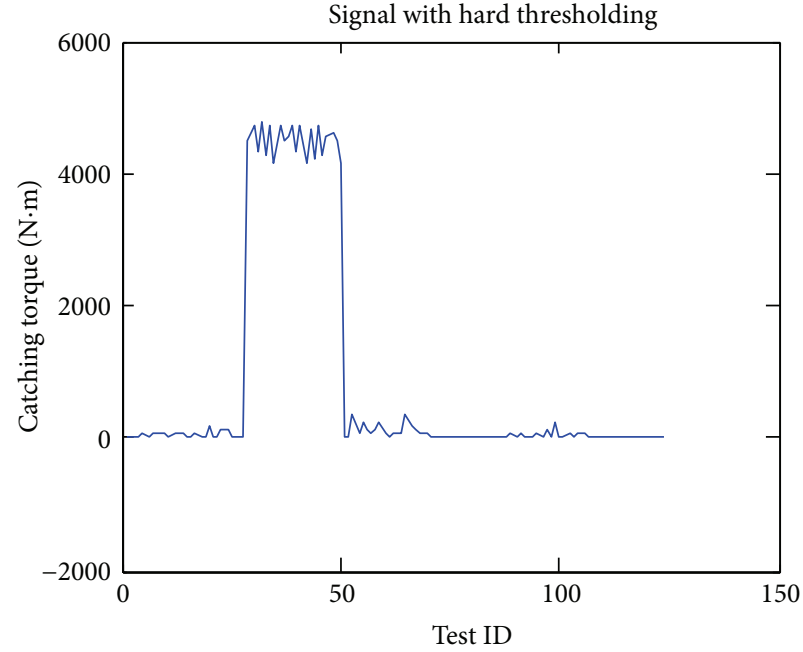

(b)

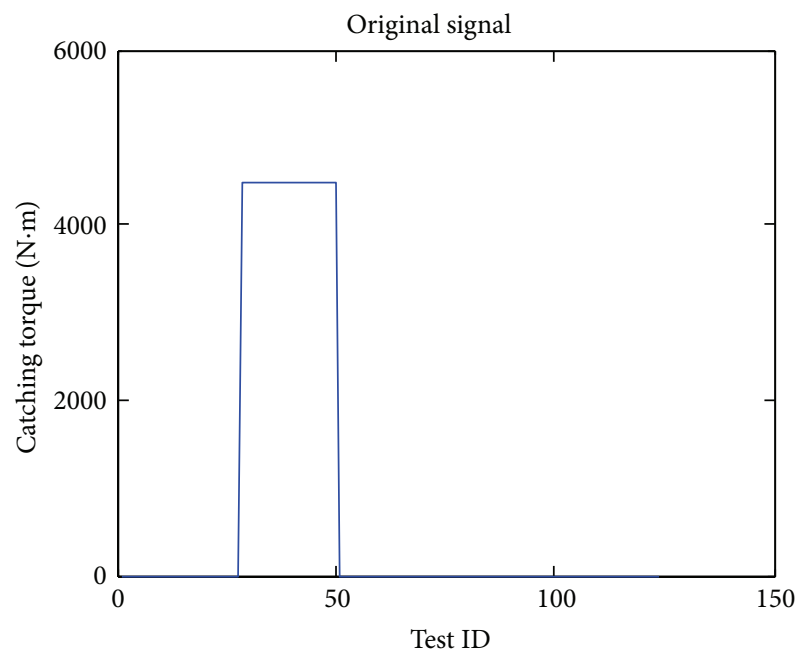

(d)

FIgURE 5: Comparisons of catching torque.

deceleration section is computed by the following equations, respectively:

$$
\begin{gathered}
v_{i 1}=v_{01}+\alpha_{1} i=142.72+(0.04+i-1) * \frac{737.78}{60}, \\
(i=1 \sim 29), \\
v_{i 2}=v_{02}+\alpha_{2} i=156-(i-29) * \frac{10173.91}{60}, \\
(i=29 \sim 52),
\end{gathered}
$$

where $v_{01}=142.72 \mathrm{rpm}, \alpha_{1}=(156-142.72) * 60 / 1.08=$ $737.78 \mathrm{rpm}^{2}, v_{02}=156 \mathrm{rpm}$, and $\alpha_{2}=-(156) * 60 /(2-1.08)=$ $-10173.91 \mathrm{rpm}^{2}$.

The original rotation speed $s_{1}$ is the assembly of $v_{i 1}$ and $v_{i 2}$; that is,

$$
s_{i}=\left\{v_{i 1}, v_{i 2}\right\} .
$$

The theoretical catching torque is computed by the following equation according to datasheet of antifalling safety device:

$$
C_{r}=(1.5 \times 8 \times 15) \times \frac{M}{2},
$$




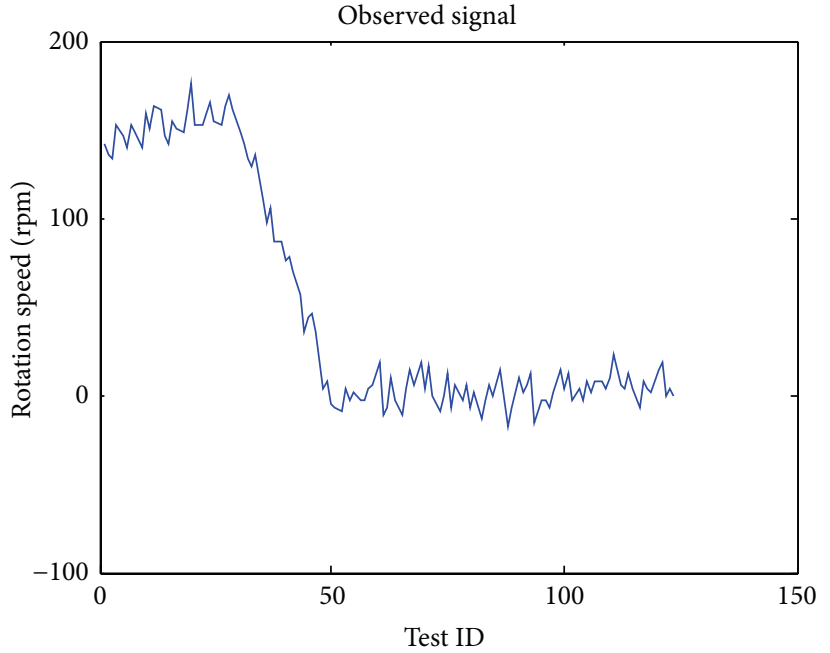

(a)

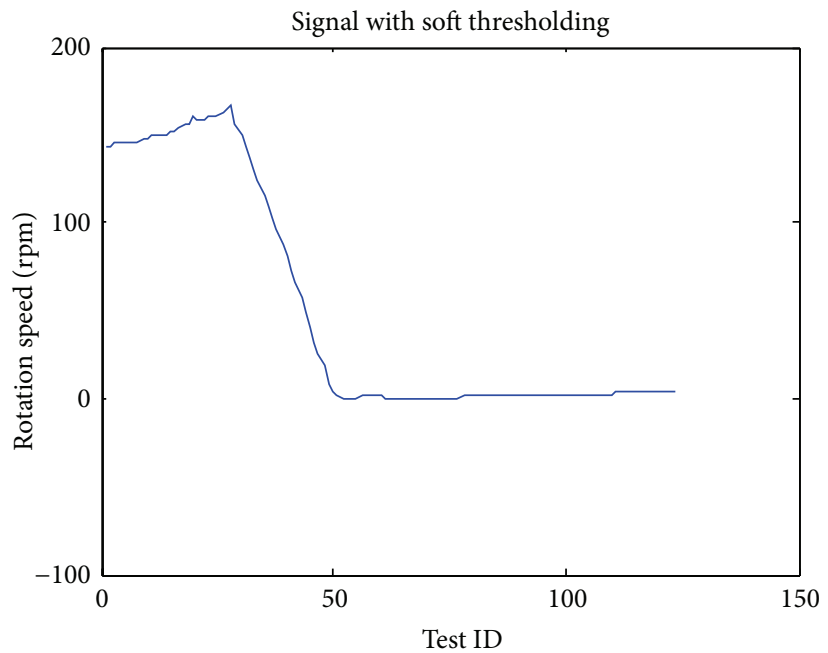

(c)

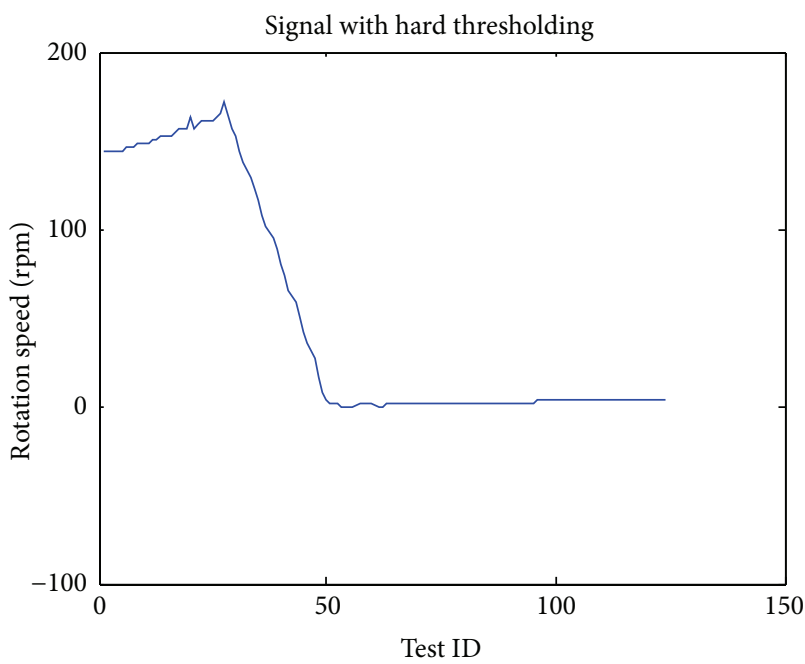

(b)

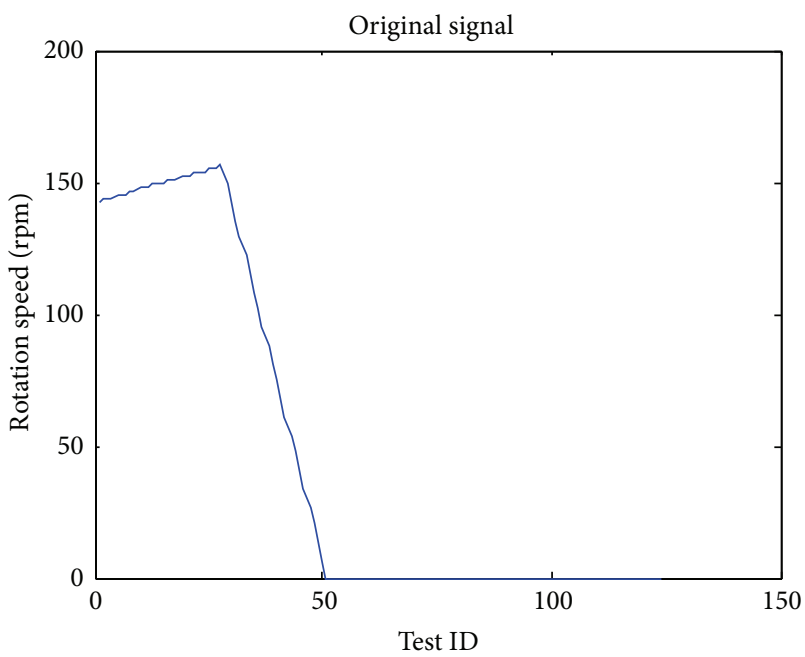

(d)

FIGURE 6: Comparisons of rotation speed.

TABLE 6: The SNR results of rotation speed with different wavelet scale coefficient $(\mathrm{dB})$.

\begin{tabular}{lccccccc}
\hline $\begin{array}{l}\text { Wavelet scale } \\
\text { coefficient }\end{array}$ & 1 & 2 & 3 & 4 & 5 & 8 & 10 \\
\hline SNR (dB) & 23.16 & 25.03 & 26.22 & 27.17 & 27.17 & 27.16 & 27.17 \\
\hline
\end{tabular}

TABLE 7: The SNR and MSE results of rotation speed with different wavelet functions of symN.

\begin{tabular}{lcccccc}
\hline & \multicolumn{6}{c}{ symN } \\
& 3 & 4 & 5 & 6 & 7 & 8 \\
\hline SNR (dB) & 26.62 & 26.71 & 26.83 & 27.10 & 27.12 & 27.17 \\
MSE & 0.5217 & 0.5072 & 0.4956 & 0.4898 & 0.4703 & 0.4699 \\
\hline
\end{tabular}

where $M$ is the rated catching load which is $44.8 \mathrm{kN}$; hence, the original catching torque $C_{r}$ is $4032 \mathrm{~N} \cdot \mathrm{m}$.
TABLE 8: The SNR and MSE of denoised signal and observed data.

\begin{tabular}{lcccc}
\hline & $\begin{array}{c}\text { Denoised } \\
\text { rotation } \\
\text { speed }\end{array}$ & $\begin{array}{c}\text { Observed } \\
\text { rotation } \\
\text { speed }\end{array}$ & $\begin{array}{c}\text { Denoised } \\
\text { catching } \\
\text { torque }\end{array}$ & $\begin{array}{c}\text { Observed } \\
\text { catching } \\
\text { torque }\end{array}$ \\
\hline SNR $(\mathrm{dB})$ & 27.17 & 26.15 & 17.33 & 16.28 \\
MSE & 0.4699 & 0.6612 & 36.7545 & 41.9621 \\
\hline
\end{tabular}

TABLE 9: Catching distance of antifalling safety device (m).

\begin{tabular}{lcccc}
\hline \multirow{2}{*}{$\begin{array}{c}\text { Number } \\
\end{array}$} & \multicolumn{3}{c}{ Compression of disc spring } & Catching distance (m) \\
\hline 1 & 23.1 & 26.8 & 3.7 & 0.697 \\
2 & 26.8 & 29.4 & 2.6 & 0.490 \\
3 & 29.4 & 31.5 & 2.1 & 0.396 \\
\hline
\end{tabular}


TABLE 10: Detected catching torque and the maximum catching load.

\begin{tabular}{lcccc}
\hline Number & $\begin{array}{c}\text { Rated catching load } \\
(\mathrm{kN})\end{array}$ & $\begin{array}{c}\text { Detected catching torque } \\
(\mathrm{kN} \cdot \mathrm{m})\end{array}$ & $\begin{array}{c}\text { Catching load measurement } \\
\text { Computed catching load } \\
(\mathrm{kN})\end{array}$ & $\begin{array}{c}\text { Maximum catching load } \\
(\mathrm{kN})\end{array}$ \\
\hline 1 & 44.8 & 3.028 & 33.644 & 50.322 \\
2 & 44.8 & 3.870 & 43.000 & 50.322 \\
3 & 44.8 & 4.529 & \\
\hline
\end{tabular}

TABLE 11: The computed results with wavelet transform denoised data.

\begin{tabular}{|c|c|c|c|c|c|}
\hline Number & $\begin{array}{c}\text { Tripping rotation } \\
\text { speed }(\mathrm{rpm})\end{array}$ & $\begin{array}{c}\text { Catching torque } \\
(\mathrm{Nm})\end{array}$ & $\begin{array}{c}\text { Spring } \\
\text { compression }(\mathrm{mm})\end{array}$ & $\begin{array}{c}\text { Catching } \\
\text { distance }(\mathrm{m})\end{array}$ & $\begin{array}{c}\text { Catching load } \\
(\mathrm{kN})\end{array}$ \\
\hline \multirow{2}{*}{101585} & 164.95 & 4819 & 3.6 & 0.679 & 53.544 \\
\hline & 157.98 & 4425 & 2.3 & 0.434 & 49.167 \\
\hline \multirow{2}{*}{101586} & 160.06 & 4461 & 1.8 & 0.339 & 49.567 \\
\hline & 157.28 & 3839.2 & 5.1 & 0.96 & 42.661 \\
\hline \multirow{2}{*}{101587} & 155.56 & 3537.8 & 5.5 & 1.037 & 39.309 \\
\hline & 156.42 & 3811.4 & 5.1 & 0.961 & 42.349 \\
\hline \multirow{2}{*}{101588} & 157.41 & 3793.6 & 5.2 & 0.980 & 42.151 \\
\hline & 157.46 & 3834.2 & 5.1 & 0.961 & 42.602 \\
\hline \multirow{2}{*}{101589} & 157.49 & 3383.4 & 5.7 & 1.074 & 37.593 \\
\hline & 157 & 3443.8 & 5.6 & 1.056 & 38.264 \\
\hline \multirow{2}{*}{101590} & 155.35 & 3544.9 & 5.5 & 1.037 & 39.388 \\
\hline & 157.43 & 3815.6 & 5.1 & 0.96 & 42.396 \\
\hline \multirow{2}{*}{101591} & 155.31 & 3659.9 & 5.4 & 1.02 & 40.666 \\
\hline & 157.31 & 3581.6 & 5.4 & 1.02 & 39.796 \\
\hline \multirow{2}{*}{101592} & 157.49 & 3363.8 & 5.8 & 1.09 & 37.376 \\
\hline & 154.19 & 3478.7 & 5.5 & 1.04 & 38.652 \\
\hline \multirow{2}{*}{101593} & 150.96 & 3383.6 & 5.7 & 1.07 & 37.60 \\
\hline & 150.43 & 3589.1 & 5.4 & 1.02 & 39.88 \\
\hline \multirow{2}{*}{101594} & 151.92 & 3622.9 & 5.4 & 1.02 & 40.25 \\
\hline & 144.88 & 3413 & 5.6 & 1.06 & 37.92 \\
\hline
\end{tabular}

A denoising signal method using soft heuristic thresholding at level 4 by sym 8 wavelet is proposed in this paper, in which an improved threshold value is given by (9).

Two thresholding methods hard thresholding and soft thresholding are compared from level 1-10 by sym 8 wavelet. The SNR results of denoised rotation speed of two thresholding option are shown in Table 5. It is shown in this table that the maximum SNR is obtained when using soft thresholding at level 4.

The SNR of rotation speed signal with different wavelet scale coefficient $j(j=1,2,3,4,5,8,10)$ by soft thresholding is shown in Table 6. According to this table, when wavelet scale coefficient $j$ is from 4 to 10 , an improved SNR of about $27.17 \mathrm{~dB}$ is achieved.

It can be shown in Table 7 that sym 8 is better than sym 3 to sym7. This can be explained by the fact that the wavelet basis with higher order approximation can achieve better denoising results than that of lower order approximation.
On the other hand, the higher order approximation may cause the original signal vanished. Therefore the actual wavelet basis has to be ensured by different experiments.

The SNRs of rotation speed signal with different wavelet functions of $\operatorname{sym} N(N=3,4, \ldots, 8)$ at level 4 are listed in Table 7.

The SNR results of the denoised signal with soft thresholding and the observed data are compared at level 4 by sym 8 wavelet and the results are shown in Table 8.

From Tables 7 and 8 , it is shown that the MSE using a soft thresholding at level 4 by sym 8 wavelet is smaller than that by other sym $N$ and in all cases smaller than MSE using hard thresholding. The SNR using sym8 wavelet is the highest among different wavelet functions of symN. Moreover, SNR of denoised signal is higher than the detected signal. In summary, the performance of the denoising method of this paper is improved compared to that of hard thresholding. Additionally, the denoising method with soft thresholding at 


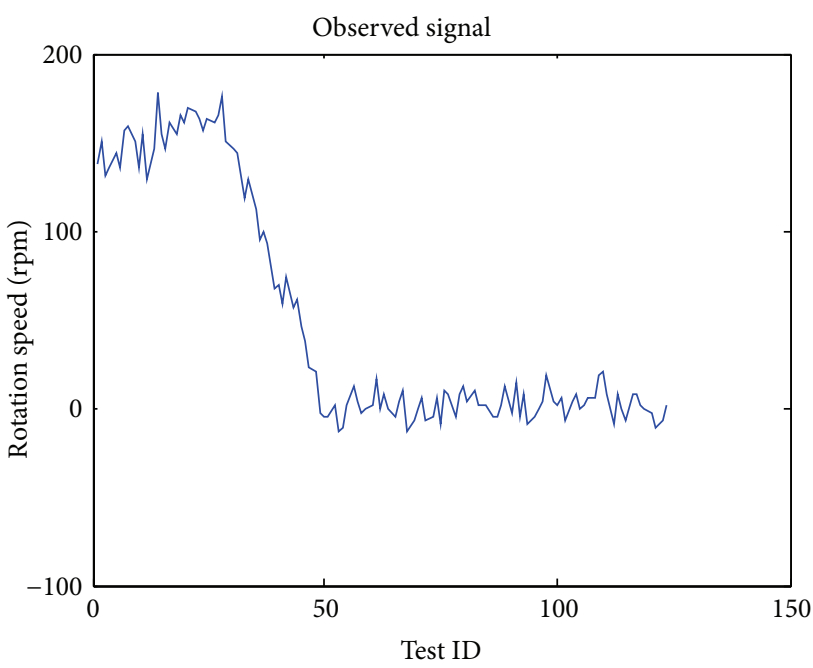

(a)

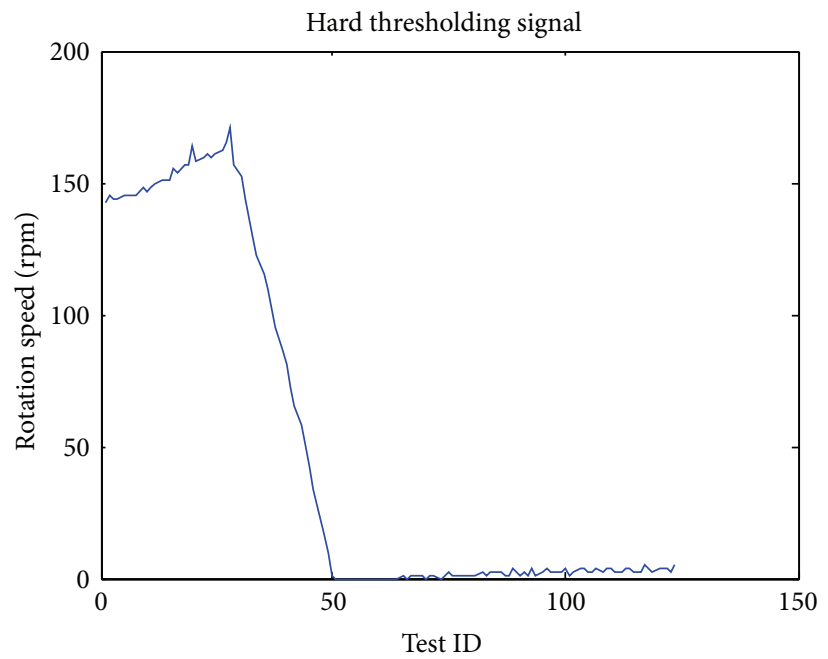

(c)

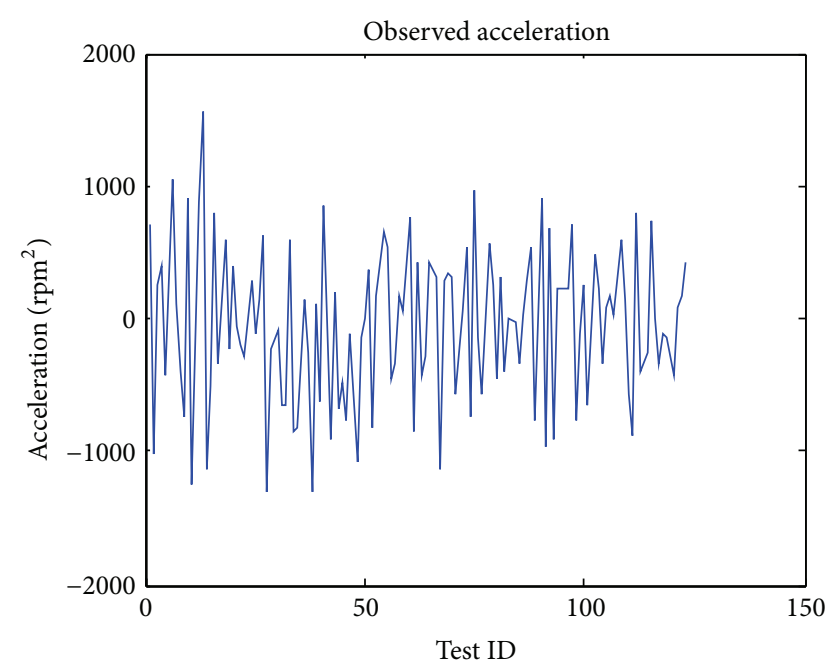

(b)

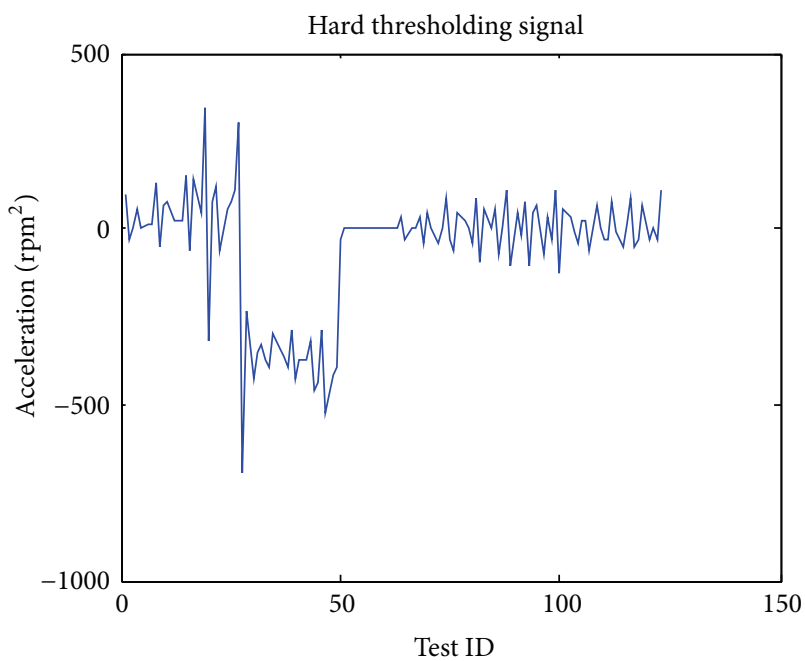

(d)

FIGURE 7: Comparisons of rotation speed and acceleration.

level 4 by sym 8 wavelet achieves a better SNR and MSE than that at other level by sym3 to sym7. The SNR of denoised rotation speed and catching torque is increased from $26.15 \mathrm{~dB}$ to $27.17 \mathrm{~dB}$ which is $3.9 \%$ improvement. Similarly, $6.4 \%$ improvement of SNR of denoised catching torque is achieved.

With the wavelet transform, the data are smoothed and the errors are reduced. The figures show certain noise suppression, the denoised catching torque, and rotation speed have a relatively small defect and signal amplitude loss.

The computed catching distance is shown in Table 9.

It is specified in Chinese specification GB/T10054 that the maximum catching distance must be less than $2 \mathrm{~m}$. Therefore, the diagnosed results in Table 9 show that the safety device is qualified.

The examples of the catching torque and the maximum catching load are shown in Table 10.

Ten groups of data of the original catching torque and the denoised catching torque with wavelet transform during the industrial detection process are selected randomly and shown in Figure 8. From this figure, it can be observed that the computation results with observed signal distribute much loosely, which may confuse the operator and the customer. When the signal is denoised, the results are closer which is more reliable.

Ten groups of denoised data with wavelet transform for computation and diagnosis are shown in Table 11. In this table, column "number" represents the ID of an antifalling safety device. Each device is tested twice. Signals in column "tripping rotation speed" and column "catching torque" are denoised by wavelet transform. The data in "spring compression" is measured manually. Catching distance column is obtained with (12) and catching load with (13).

Based on the denoising signal method using thresholding with wavelet transform, the catching load of the antifalling safety device is more accurate and reliable than the observed signals. 


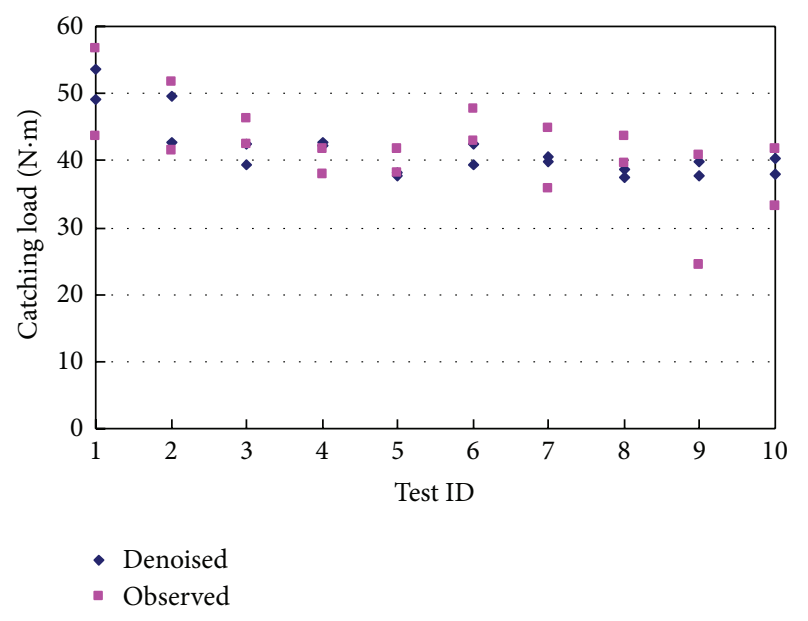

FIGURE 8: Catching load comparisons of observed signal and denoised signal.

\section{Conclusions}

Condition diagnosis of an antifalling safety device depends largely on the rotation speed and catching torque signals measured on the platform because the signals carry dynamic information about the machine state. However, the measured signal for fault diagnosis is difficult because the signal contains strong noise. Noise in the actual signal may lead to misrecognition and unreliability of useful information for the condition diagnosis. To solve these problems, we proposed a wavelet based condition diagnosis method for antifalling safety device. A noise reduction method employing hard and soft thresholding of DWT is proposed to denoise two important signals in the condition diagnosis. The wavelet transform with thresholding shows a better result than the measured rotation speed and catching torque signals. The experimental result shows that the signals are smoothed and the errors are reduced. An averagely maximum data error reduction of $7.5 \%$ is obtained. SNRs are improved by $3.9 \%$ and $6.4 \%$ for rotation speed and catching torque, respectively. The denoised signals improve the accuracy and reliability of diagnosis result for the antifalling safety device in construction elevator. The safety of elevator is ensured in high-rise building construction.

\section{Conflict of Interests}

The authors declare that there is no conflict of interests regarding the publication of this paper.

\section{Acknowledgments}

This work is partially funded by Chongqing Technology and Business University through a Young Scholar seed Grant (Grant no. 1352015). The experiment environment and the antifalling safety device are provided by Chongqing Yingfengsheng Machinery and Equipment Co., Ltd. The authors thank for the efforts made by Professor Chen Shijiao,
Mr. Chen Yong, and Mr. Wang Hongyi during the experimental process.

\section{References}

[1] G. Tian, "Limit range of braking distance for anti-falling device with gear conedrum," Construction Machinery, vol. 2, pp. 25-28, 2000.

[2] X.-J. Hao, "The analysis of auto detecting the brake of elevator using numerical arithmetic," Microcomputer Information, vol. 25, no. 5, pp. 310-312, 2009.

[3] B. Zhang, T. Luan, and T. Ma, "On the importance of inspection of construction hoist anti falling safety device," Value Engineering, vol. 6, pp. 96-97, 2013.

[4] P. J. Tavner, "Review of condition monitoring of rotating electrical machines," IET Electric Power Applications, vol. 2, no. 4, pp. 215-247, 2008.

[5] J. Bai and J. Zhang, "Developing of anti-falling safety device detection system of construction hoist based on VB," Gansu Science and Technology, vol. 28, no. 7, pp. 53-55, 2012.

[6] K. Li, P. Chen, and H. Wang, "Intelligent diagnosis method for rotating machinery using wavelet transform and ant colony optimization," IEEE Sensors Journal, vol. 12, no. 7, pp. 24742484, 2012.

[7] S. Venugopal, R. A. Wagstaff, and J. P. Sharma, "Exploiting phase fluctuations to improve machine performance monitoring," IEEE Transactions on Automation Science and Engineering, vol. 4, no. 2, pp. 153-166, 2007.

[8] H. Sun, K. Li, P. Chen, H. Wang, X. Ping, and Y. Cao, "A sequential fuzzy diagnosis method for rotating machinery using ant colony optimization and possibility theory," Journal of Mechanical Science and Technology, vol. 28, no. 4, pp. 1189-1201, 2014.

[9] J. G. Mueller and T. G. Pratt, "A radio frequency polarimetric sensor for rotating machine analysis," IEEE Sensors Journal, vol. 13, no. 12, pp. 4866-4873, 2013.

[10] S. S. Tayarani-Bathaie, Z. N. S. Vanini, and K. Khorasani, "Dynamic neural network-based fault diagnosis of gas turbine engines," Neurocomputing, vol. 125, pp. 153-165, 2014.

[11] Z. Yang, B. W.-K. Ling, and C. Bingham, "Fault detection and signal reconstruction for increasing operational availability of industrial gas turbines," Measurement, vol. 46, no. 6, pp. 19381946, 2013.

[12] Y. Kim, J. W. Chong, K. H. Chon, and J. Kim, "Wavelet-based AR-SVM for health monitoring of smart structures," Smart Materials and Structures, vol. 22, no. 1, Article ID 015003, pp. 1-12, 2013.

[13] K. V. Nguyen, "Comparison studies of open and breathing crack detections of a beam-like bridge subjected to a moving vehicle," Engineering Structures, vol. 51, pp. 306-314, 2013.

[14] D. L. Donoho, "De-noising by soft-thresholding," IEEE Transactions on Information Theory, vol. 41, no. 3, pp. 613-627, 1995.

[15] J. Li, B. Yu, and H. Fischer, "Wavelet transform based on the optimal wavelet pairs for tunable diode laser absorption spectroscopy signal processing," Applied Spectroscopy, vol. 69, no. 4, pp. 496-506, 2015.

[16] C. Li, M. Liang, and T. Wang, "Criterion fusion for spectral segmentation and its application to optimal demodulation of bearing vibration signals," Mechanical Systems and Signal Processing, vol. 64-65, pp. 132-148, 2015. 
[17] C. Li and M. Liang, "Enhancement of oil debris sensor capability by reliable debris signature extraction via wavelet domain target and interference signal tracking," Measurement, vol. 46, no. 4, pp. 1442-1453, 2013.

[18] K. Dziedziech, W. J. Staszewski, and T. Uhl, "Wavelet-based frequency response function: comparative study of input excitation," Shock and Vibration, vol. 2014, Article ID 502762, 11 pages, 2014.

[19] M. Singh and R. Kumar, "Thrust bearing groove race defect measurement by wavelet decomposition of pre-processed vibration signal," Measurement, vol. 46, no. 9, pp. 3508-3515, 2013.

[20] V. Muralidharan and V. Sugumaran, "Rough set based rule learning and fuzzy classification of wavelet features for fault diagnosis of monoblock centrifugal pump," Measurement, vol. 46, no. 9, pp. 3057-3063, 2013.

[21] P. Rajeev and K. K. Wijesundara, "Energy-based damage index for concentrically braced steel structure using continuous wavelet transform," Journal of Constructional Steel Research, vol. 103, pp. 241-250, 2014.

[22] J. Li, B. Yu, W. Zhao, and W. Chen, "A review of signal enhancement and noise reduction techniques for tunable diode laser absorption spectroscopy," Applied Spectroscopy Reviews, vol. 49, no. 8, pp. 666-691, 2014.

[23] A. Azzalini, M. Farge, and K. Schneider, "Nonlinear wavelet thresholding: a recursive method to determine the optimal denoising threshold," Applied and Computational Harmonic Analysis, vol. 18, no. 2, pp. 177-185, 2005.

[24] D. L. Donoho and I. M. Johnstone, "Ideal spatial adaptation by wavelet shrinkage," Biometrika, vol. 81, no. 3, pp. 425-455, 1994.

[25] D. L. Donoho, "Nonlinear wavelet methods for recovery of signals. Densities, and spectra from indirect and noisy data," in Proceedings of Symposia in Applied Mathematics, vol. 47, pp. 173205, American Mathematical Society, 1993.

[26] D. L. Donoho, "Unconditional bases are optimal bases for data compression and for statistical estimation," Applied and Computational Harmonic Analysis, vol. 1, no. 1, pp. 100-115, 1993.

[27] V. A. Tran, S. T. Quek, and W. H. Duan, "Sensor validation in damage locating vector method for structural health monitoring," International Journal of Structural Stability and Dynamics, vol. 11, no. 1, pp. 149-180, 2011.

[28] N. Wu and Q. Wang, "Experimental studies on damage detection of beam structures with wavelet transform," International Journal of Engineering Science, vol. 49, no. 3, pp. 253-261, 2011.

[29] S.-T. Quek, Q. Wang, L. Zhang, and K.-K. Ang, "Sensitivity analysis of crack detection in beams by wavelet technique," International Journal of Mechanical Sciences, vol. 43, no. 12, pp. 2899-2910, 2001.

[30] Q. Wang and N. Wu, "Detecting the delamination location of beam with a wavelet transform: an experimental study," Smart Materials and Structures, vol. 20, no. 1, Article ID 012002, 2011.

[31] Q. Wang and X. Deng, "Damage detection with spatial wavelets," International Journal of Solids and Structures, vol. 36, no. 23, pp. 3443-3468, 1999.

[32] S. Mallat and W. L. Hwang, "Singularity detection and processing with wavelets," IEEE Transactions on Information Theory, vol. 38, no. 2, pp. 617-643, 1992.

[33] D. T. Nguyen and S. T. Quek, "Reliability of switched modelbased controller for vessel dynamic positioning with switching under estimated motion frequency," Journal of Offshore Mechanics and Arctic Engineering, vol. 132, no. 2, pp. 1-9, 2010.
[34] I. Daubenchies, Ten Lectures on Wavelets, SIAM, Philadelphia, Pa, USA, 1994.

[35] H. Wen and Z. Zhang, "Improvement of denoising of nonlinear wavelet transform threshold value method," Bulletin of Surveying and Mapping, vol. 3, pp. 318-321, 2006.

[36] Z. Ruizhen and S. Guoxiang, "An improved method for white noise reduction based on wavelet transform," Journal of Xidian University, vol. 27, no. 5, pp. 619-622, 2000.

[37] J. J. G. de la Rosa, I. Lloret, A. Moreno, C. G. Puntonet, and J. M. Górriz, "Wavelets and wavelet packets applied to detect and characterize transient alarm signals from termites," Measurement, vol. 39, no. 6, pp. 553-564, 2006.

[38] M. E. Alexander, R. Baumgartner, A. R. Summers et al., "A wavelet-based method for improving signal-to-noise ratio and contrast in MR images," Magnetic Resonance Imaging, vol. 18, no. 2, pp. 169-180, 2000.

[39] P. S. Gokhale, "ECG signal De-noising using discrete wavelet transform for removal of $50 \mathrm{~Hz}$ PLI noise," International Journal of Emerging Technology and Advanced Engineering, vol. 2, no. 5, pp. 81-87, 2012. 

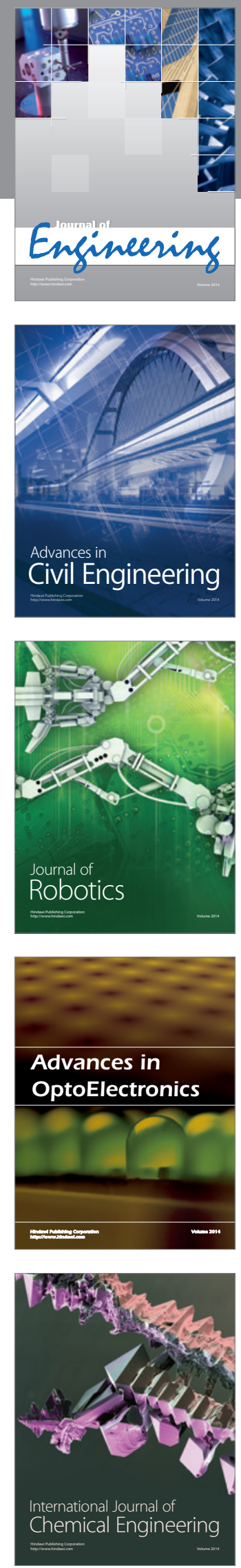

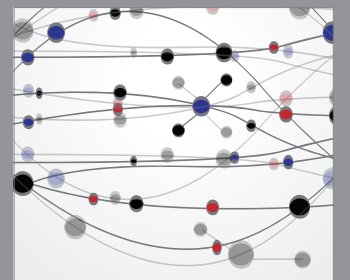

The Scientific World Journal
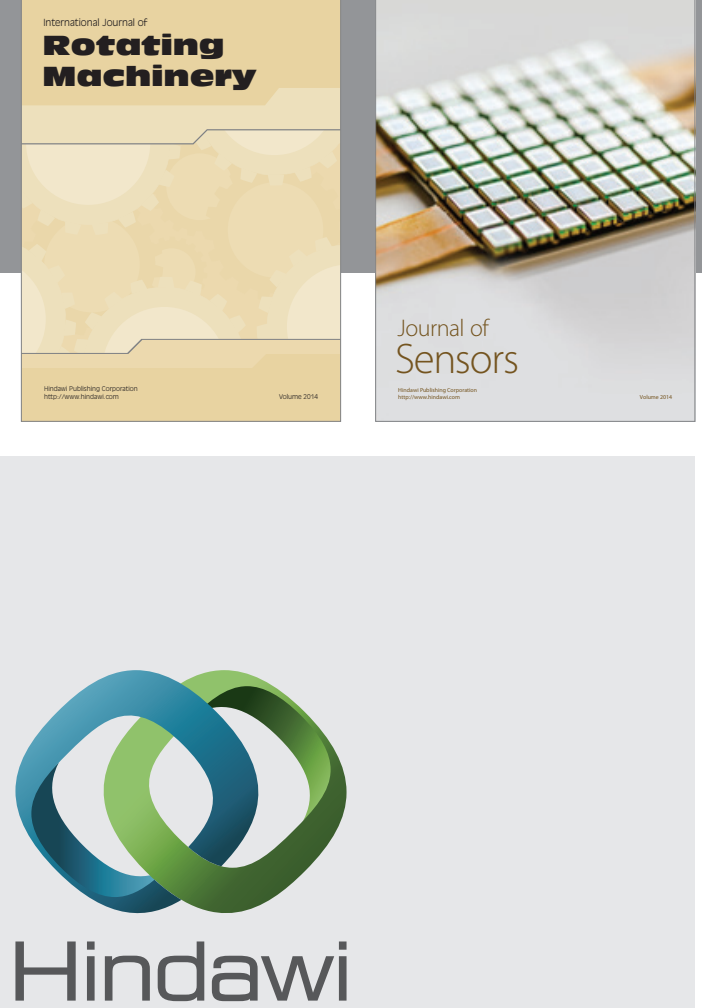

Submit your manuscripts at http://www.hindawi.com
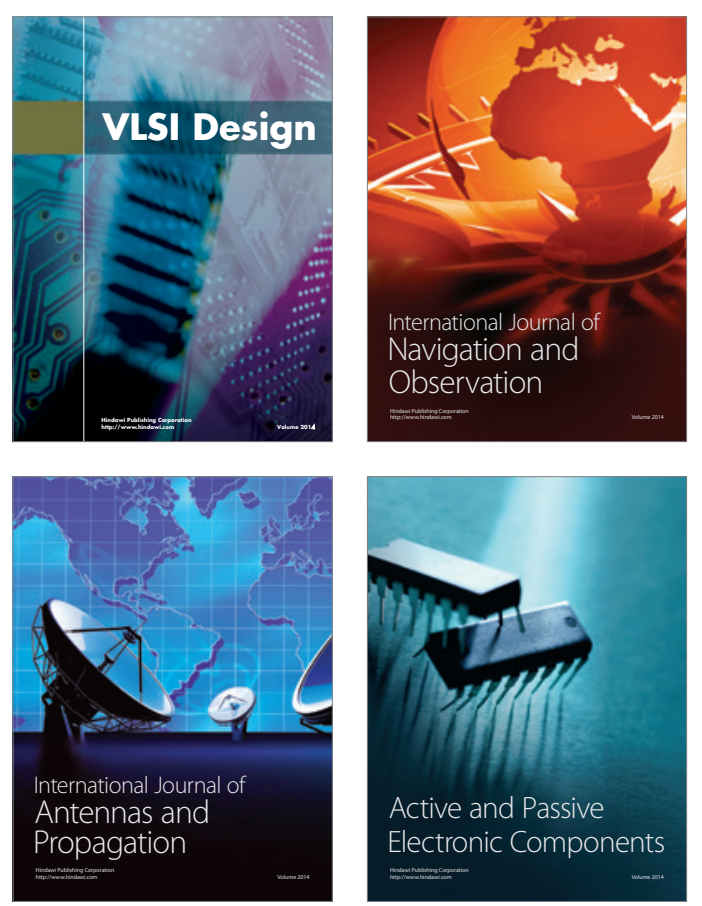
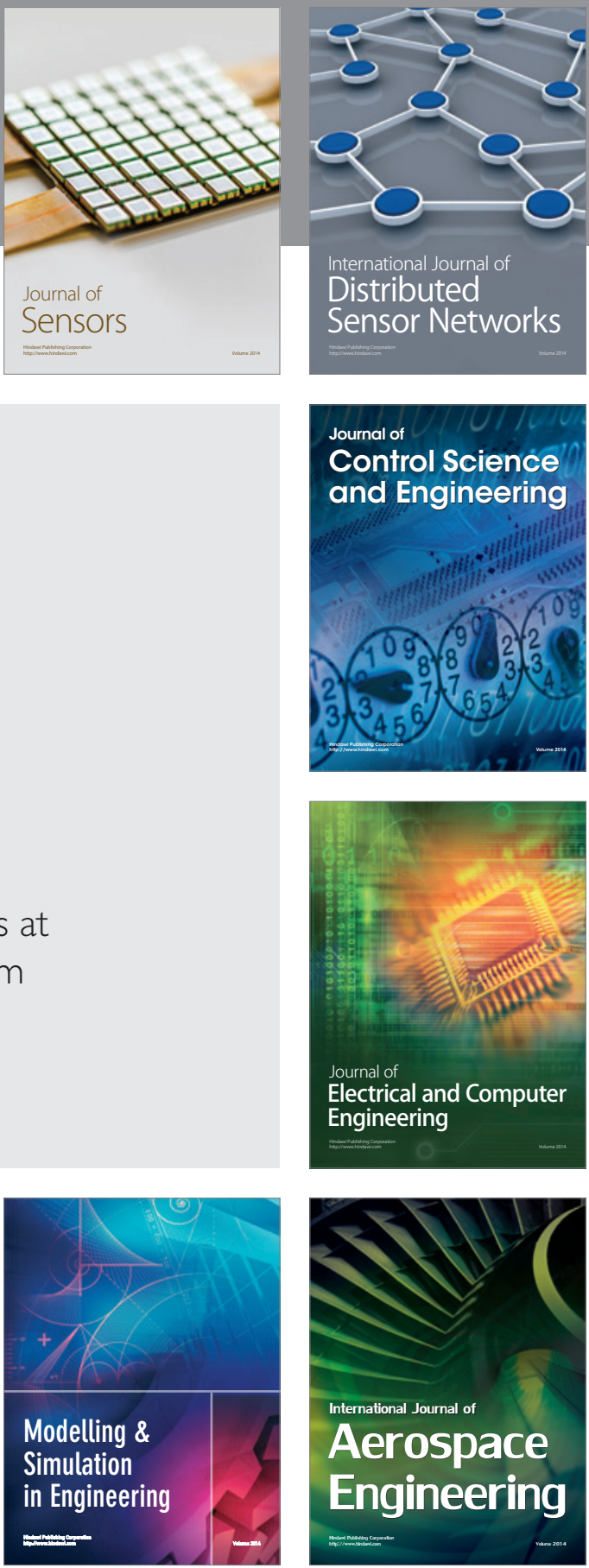

Journal of

Control Science

and Engineering
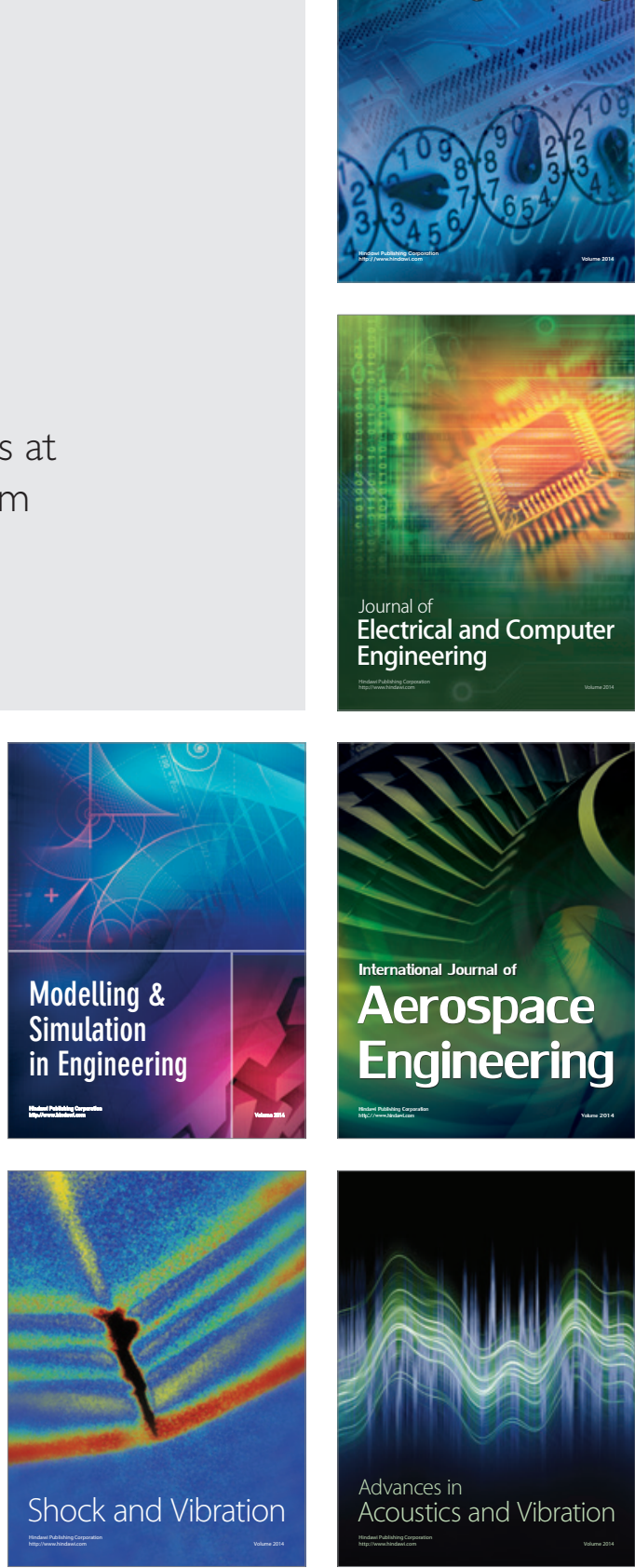